\title{
Prey speed up, predators slow down: non-consumptive effects on movement behavior of a ciliate predator-prey pair
}

\author{
Uriah Daugaard $^{1}$, Reinhard Furrer ${ }^{2}$, and Owen L. Petchey ${ }^{1}$ \\ ${ }^{1}$ Department of Evolutionary Biology and Environmental Studies, University of Zurich, \\ Winterthurerstrasse 190, 8057 Zurich, Switzerland \\ ${ }^{2}$ Department of Mathematics and Computational Science, University of Zurich, \\ Winterthurerstrasse 190, 8057 Zurich, Switzerland
}

ORCID of authors:

- Uriah Daugaard: 0000-0003-0679-1045

- Reinhard Furrer: 0000-0002-6319-2332

- Owen Petchey: 0000-0002-7724-1633

Article type: Research Article

Word counts: 244 in abstract; 122 in significance statement; 5151 in main text; 446 in captions

Number of references: 73

\section{Keywords}

10 Predator-prey interactions, foraging, behavioral plasticity, protist, anti-predator defenses, movement ecology 


\section{Abstract}

\section{Significance Statement}

Non-consumptive effects (NCEs) of predators on prey, such as induced defensive strategies, are frequently neglected in the analysis of predator-prey interactions. Yet these effects can have demographic impacts as strong as consumption. As a counterpart to NCEs, resource-availability effects (RAEs) can prompt changes in predators as well, e.g., in their foraging behavior. We studied NCEs and RAEs in the ciliate predator-prey pair Didinium nasutum and Paramecium caudatum. We examined the dependence of prey/predator swimming speed and body size on predator/prey presence. We also investigated prey spatial grouping behavior and the dependence of predator movement on local prey abundance. We collected individual movement and morphology data through videography of laboratory-based populations. We compared swimming speeds and body sizes based on their distributions. We used linear models to respectively quantify the effects of local prey abundance on predator displacements and of predator presence on prey grouping behavior. In the presence of prey, predator individuals swam more slowly, were bigger and made smaller displacements. Further, their displacements decreased with increasing local prey abundance. In contrast, in the presence of predators, proportionally more prey individuals showed a fast-swimming behavior and there was weak evidence for increased prey grouping. Trait changes entail energy expenditure shifts, which likely affect interspecific interactions and populations, as has been shown for NCEs. Less is known about the link between RAEs and demography, but it seems likely that the observed effects scale up to influence community and ecosystem stability, yet this remains largely unexplored.

To maximize their fitness, organisms balance investment in foraging and avoiding being eaten. The behaviors of prey and predators are thus expected to depend on the presence and absence of each other and serve either to boost the chances of predation evasion or to increase predation success. Here we provide an example of the co-dependence of behaviors in the predator-prey pair Didinium nasutum and Paramecium caudatum. We show that the predator slows down and searches in smaller areas when prey are present, while the prey speeds up and possibly groups more as a response to the presence of predators. Such behavioral changes are likely to have a demographic and community impact that is not accounted for with common measures of predators-prey interactions. 


\section{Introduction}

Predation is an ubiquitous interspecific interaction and can contribute to the long-term coexistence of species (MacArthur 1984; Chesson 2000). Keystone species are known to maintain biodiversity (Paine 1969; Mills et al. 1993) while more recent results suggest that weak consumer-resource interactions can stabilize a food web containing multiple strong interactions (O’Gorman and Emmerson 2009; Kadoya et al. 2018).

In addition to consuming their prey, predators also have non-consumptive effects (NCEs, also known as trait-mediated interactions and as non-lethal effects). These include inducing defensive strategies and stress-induced changes in behavior (Lima 1998; Werner and Peacor 2003; Hermann and Landis 2017; MacLeod et al. 2018). For the prey, these changes can lead to a decreased energy intake, increased energy expenditure for the defense against predation, and decreased growth rates (e.g. Preisser et al. 2005; Clinchy et al. 2013). These effects scale up to have an impact on prey demography that can be at least as strong as the one caused by direct consumption (Preisser et al. 2005). NCEs are thus hypothesized to affect community stability (Anholt and Werner 1995; Peckarsky et al. 2008), with some evidence that this is the case (van Veen et al. 2005; Fill et al. 2012)

Similarly to the prey reacting to predator-presence, predators are expected to adapt their traits to the abundance of their prey. Here, we refer to this dependency as resource availability effects (RAEs), which can be seen as trait mediated interactions from the prey to the predator. For instance, predators can increase their foraging efforts when prey are scarce (Munk 1995; Ronconi and Burger 2008). In general, predator characteristics such as optimal patch use (e.g. McNamara 1982; Wajnberg et al. 2006) and optimal diet and foraging (O’brien et al. 1990; Sih and Christensen 2001) are likely to depend on prey abundance (i.e. resource availability). As an example of the prey density dependence of these processes, a simple Gaussian random walk is sufficient as a search strategy for the foraging of abundant prey, while a more efficient search strategy becomes increasingly important with decreasing resource availability (Humphries et al. 2010).

A predator-prey interaction is thus defined by the consumptive and the non-consumptive effects of the predator on the prey and by the resource availability effects of the prey on the predator. Yet, predator-prey interaction strengths are frequently calculated by only considering predator feeding rates as a function of prey abundance (i.e. the functional response Berlow et al. 2004; Rall et al. 2010; Sentis et al. 2012; Kalinoski and DeLong 2016; Daugaard et al. 2019). 
61 The functional response represents the mortality rate of the prey due to the consumption by the predator. Its product with the conversion efficiency is often used in models as the increase rate of the predator population (e.g. Yodzis 1994; Abrams and Ginzburg 2000). In other words, this approach does not account for any NCEs and RAEs impacting the growth rate of the prey (Van Buskirk and Yurewicz 1998; Preisser and Bolnick 2008; Sheriff et al. 2020) and the mortality rate and conversion efficiency of the predator (Bozer et al. 1996; Minter et al. 2011; Li and Montagnes 2015). To thoroughly characterize such a predator-prey pair, it is thus not enough to limit the analysis to the functional response (Lima 1998; Schmitz 2017).

Here we investigated the presence and characteristics of NCEs and RAEs in a microbial predator-prey pair of the ciliates Didinium nasutum (predator) and Paramecium caudatum (prey). Due to their favorable traits (e.g. short generation times and low maintenance) ciliates are frequently used in laboratory-based experiments for gaining insights into processes and patterns that are difficult to discern otherwise (Altermatt et al. 2015). Indeed, some of the earliest experiments about predator-prey dynamics were performed with Didinium and Paramecium (Luckinbill 1974). While simple (i.e., unicellular organisms without a nervous system), ciliates display various reactions to their environment such as bending, ciliary alteration, contractions and detachment (Jennings 1902; Dexter et al. 2019). Prey ciliate species have been observed to significantly reduce their swimming speed, increase their body width and to show additional defenses, such as flight upon encounter, as a reaction to the presence of predators (Kusch 1993; Broglio et al. 2001; Hammill et al. 2009, 2010; Wu et al. 2010). Moreover, resource-dependent morphology has been observed in predatory ciliate species, as they changed their cell volume in response to the size of their prey, and thereby increased the probability of a successful attack (Hewett 1980, 1988). Because of this existing evidence of RAEs and NCEs and the possibility of collecting individual-based morphology, movement and location data through videography, ciliate predator-prey pairs thus offer a convenient and informative system to further study and describe RAEs and NCEs.

We tested for NCEs on prey swimming speed and spatial grouping behavior, hypothesizing that in the presence of the predator the prey would swim faster to increase evasion rates, and would group more and increase in body size to decrease the per capita predation risk. Regarding RAEs, we investigated predator body size, swimming speed and diffusion in absence and presence of prey, and the dependence of predator movement on local prey scarcity. We 
87

88

\section{$89 \quad$ Material and Methods}

\section{$90 \quad$ Material and Experiment}

91 We carried out an experiment to observe the behavior of ciliates Didinium nasutum (predator) and Paramecium

consequence of swimming faster and straighter) in the absence of prey and to perform bigger displacements the fewer prey individuals are in its proximity to increase the encounter rate with prey. caudatum (prey) in absence and presence of each other. Before and during the experiment, we kept the ciliates in bacterized organic protozoan pellet medium (Carolina Biological Supply Company, Burlington NC; concentration of $0.55 \mathrm{~g} \mathrm{~L}^{-1}$, see Altermatt et al. 2015) at $15{ }^{\circ} \mathrm{C}$. The predator was kept in several maintenance six-well plates and fed with P. Caudatum ad libitum.

The experimental units were Petri dishes (diameter of $5.4 \mathrm{~cm}$ ) each containing $3 \mathrm{~mL}$ of bacterized medium and only $P$. caudatum individuals (prey monoculture), only D. nasutum individuals (predator monoculture) or both species (mixed culture), with four replicates for each of these three compositions. From the maintenance plates, we randomly selected 60 predator individuals and manually pipetted them to each replicate of the predator monoculture and of the mixed culture. We confirmed the number of predator individuals under a light microscope after the transfer. In the case of the mixed and the prey monoculture we added approximately 400 prey individuals per milliliter. We incubated the replicates for four hours in the dark to give the individuals time to reinstate any behavioral and spatial dependency that might have been disturbed during the preparation of the experimental units.

After the incubation time, we recorded three 30 seconds videos of each replicate at magnification 12.5 with 25 frames per second (750 frames per video) using a Hamamatsu C11440 camera, a Leica M205 C dissecting microscope with dark field illumination, and the software HCImage Live. We had an interval between videos of the same Petri dish of one minute, to ensure a greater independence of the videos as individuals could move in and out of the frame in that time. The videos covered a square of side-length $1.7 \mathrm{~cm}$ (i.e., an area of $2.89 \mathrm{~cm}^{2}, 12.6 \%$ of the surface of the Petri dish) in the center of the Petri dish. The captured area within the Petri dishes was the same across all experimental units and data collection was blinded. 
111 We analyzed the videos with the R-package bemovi (Pennekamp et al. 2015), which tracks particles and extracts their

112 trajectory and morphology data. To ensure data quality, we only analyzed trajectories that were at least two seconds

113 long. We determined species identities of the tracked particles in the mixed culture replicates with a random forest 114 classification $(0.04 \%$ out-of-bag estimate of the error rate).

\section{Data Analysis}

116 We used various variables to investigate the research questions and hypotheses. These variables, their calculations and 117 use are described below and summarized in Table 1.

\section{Swimming speed and body size}

The recorded videos were two-dimensional, providing the $(x, y)$-coordinates of the individuals across time. However, while thin (i.e. $1.31 \mathrm{~mm}$ ) the layer of medium in the Petri dishes still allowed the ciliates to move in the $z$-direction to a small extent. In fact, the predator typically swims in helices (Salt 1979), which is a three-dimensional type of movement. To account for this in the estimation of the predator swimming speed, we made use of fact that the $z$ component of the velocity of a helical trajectory is zero twice per complete helix turn, if the helix lies in the ( $x, y)$-plane (see Gurarie et al. 2011). We identified these points as the local maxima of the two-dimensional speed of a predator individual (i.e., its $(x, y)$-displacement per frame). We then estimated the swimming speed of each predator individual by taking the 60th percentile of these local maxima, as this percentile proved to be a reliable estimate of the swimming speed (see supplementary Section S2.1 for more information regarding predator swimming speed calculation). As the predator is approximately the shape of a sphere (Kahl 1930) and thus independent of its orientation, we estimated the body size of each predator individual by taking the mean of its recorded body sizes during its entire trajectory.

Unlike the predator, the prey swims in more or less straight lines. As the prey is cigar-shaped with a length of 170-290 $\mu \mathrm{m}$ and a width of 50-80 $\mu \mathrm{m}$ (based on Wichterman 2012, and personal observations), its aspect ratio is always larger than two when it swims in the $(x, y)$-plane but it can be smaller than two when it swims perpendicularly to the $(x, y)$ plane. We estimated the swimming speed and the body size of each prey individual by respectively taking the median of its swimming speed and the mean of its body size that we recorded considering only the parts of its trajectory in 
which the aspect ratio was larger than or equal to two (indicating only small or no movement in the $z$-direction, see supplementary Fig. S8).

To test for differences in swimming behavior and body size in absence and presence of their trophic opponent, for both the prey and the predator we selected suitable theoretical distributions (i.e. probability density functions) for their swimming speeds and body sizes based on the empirical distributions of these measures. We then fitted these distributions via maximum likelihood (R-package bbml, Bolker and Team 2017) and compared the estimated parameters and their corresponding 95\% confidence intervals between mixed and monocultures. For the swimming speed of the predator as well as for the body size of both species, we chose a skew normal distribution (function snorm() in R-package fGarch, Fernández and Steel 1998; Wuertz et al. 2019). In this distribution, a symmetric normal density is achieved with the skewness parameter $=1$, right- and left-skewed normal distributions respectively with skewness $>1$ and $<1$. For the prey swimming speed, we used a weighted mixture of two normal distributions, i.e. $f\left(v_{\text {prey }}\right)=$ $\omega \phi\left(v_{\text {prey }}, \mu_{1}, \sigma_{1}^{2}\right)+(1-\omega) \phi\left(v_{\text {prey }}, \mu_{2}, \sigma_{2}^{2}\right)$. In the equation, $v_{\text {prey }}$ is the swimming speed of the prey and $f\left(v_{\text {prey }}\right)$ its density function, $\phi$ is the density function of a normal distribution, $\omega$ the weight used to combine the two normal distributions and $\mu_{1}, \mu_{2}, \sigma_{1}$ and $\sigma_{2}$ respectively the means and the standard deviations of the two normal distributions. To assess the relation between swimming speed (response variable) and body size (explanatory variable) in the two species, we fitted linear models including an interaction with the culture type (mixed and monoculture).

\section{Predator displacements}

To characterize the displacements of the predator we computed the mean square displacement MSD (i.e., the average squared displacement of the predator) using the estimator $\widehat{\operatorname{MSD}}(\tau)=\left\langle|\mathbf{r}(t+\tau)-\mathbf{r}(t)|^{2}\right\rangle$ (see e.g. Ariel et al. 2015).

In the equation, $\mathbf{r}(t)$ is the location vector at time $t$ and the angle brackets indicate the averaging done first over time

(time-average) and then over the number of trajectories (ensemble-average) for a time-lag value $t$ (see for instance Janczura and Weron 2015). The MSD is further proportional to time (MSD $\propto t^{\gamma}$, see Méndez et al. 2014) with the exponent $\gamma$ indicating whether displacements follow a normal $(\gamma=1)$ or an anomalous diffusion $(\gamma \neq 1)$. 
To assess whether the predator performed larger displacements in the absence of prey we compared the $95 \%$ confidence intervals of the estimated MSD values (based on 10'000 bootstrapped replicates of the calculated time-average MSD). We investigated changes in types of diffusion with linear regression by estimating $\gamma$ as the slope in the $\log _{10}$ transformed relation between MSD and time for time-lag values in the interval from 0.32 to 9 seconds (from 8 to 225 frames). The lower bound of the interval was chosen such that the helices of the predator trajectories did not influence the MSD, and the upper bound was limited by the number of long trajectories.

We computed the number of neighbors of each predator individual as the number of predators and prey within a detection radius of $1500 \mu \mathrm{m}$. We chose this radius considering the swimming speed of the predator and carried out a sensitivity analysis to assess its impact on the analysis. To limit computational costs, we did this only for every 25 th recorded video frame. The results of the analysis were invariant to which frames were selected. We further computed the displacements that the predator individuals made in between the considered frames.

We investigated the relation between predator displacements (response variable) and prey abundances with a linear regression. The independent variables were prey abundance, predator abundance, and the culture type (i.e., predator monoculture or mixed culture) with an interaction term between the latter two. Further, to assess whether the number of neighbors (regardless of species identity) or the prey absence had a bigger impact on predator behavior, we calculated the mean number of neighbors for the predator individuals tracked in the local neighbor analysis described above. We then used this as an independent variable together with an interaction with culture type in a linear regression that had the swimming speed of the considered predator individuals as the response variable.

\section{Prey grouping}

We computed a grouping index to quantify the effect of the predator on the degree of grouping in the prey. For every 25th frame we counted the number of observed prey individuals $n$ and calculated the Euclidian nearest prey neighbor distance of each prey individual present. We then averaged the nearest prey neighbor distance within each frame to yield the mean nearest neighbor distance, denoted here as MNND.

For comparison, we estimated the uniform neighbor distance $\operatorname{UND}(n)$ (i.e. the distance between $n$ equally spaced individuals, see supplementary Fig. S13). We did this estimation by using the function rSSI() in the R-package spatstat, which places $n$ individuals in the area captured by the videos according to a given inhibition distance (the individuals 
cannot be placed closer to each other than this distance). We selected the biggest inhibition distance as the UND( $n)$ for each call after 1000 failed point placements.

We defined the grouping index GI as GI(n) $=1-\operatorname{MNND} / \mathrm{UND}(n)$ and calculated it for each considered frame. By dividing MNND by $\operatorname{UND}(n)$, the index is standardized for the different number of individuals $n$ present in the frames. The resulting index can have values between 0 (no grouping, the individuals have an inhibition distance) and 1 (complete grouping, all individuals are in one point) and accounts for different number of individuals present, which means that GI is comparable across frames.

193 We used the grouping index GI as the response variable in a normal mixed effects model with the culture type (mixed and monoculture), time and their interaction as fixed effects and video nested in sample as random intercepts. As a test of robustness, we repeated this analysis with a different measure of prey grouping as the response variable (based on the intensity functions of prey spatial point patterns, see supplementary Section S4). Further, the analysis was robust to the selection of the frames considered.

\section{Results}

199 We tracked a total of 11423 trajectories that were at least two seconds long. Of these, $14.7 \%$ belonged to predator 200 individuals and $85.3 \%$ to prey individuals. Most trajectories were shorter than 10 seconds (Fig. S1). After the incubation period of four hours, there were fewer prey cells in the mixed culture replicates than in the prey monoculture replicates, suggesting either predation of $P$. caudatum by $D$. nasutum or decreased growth rate of the prey in presence of the predator, or both (Fig. S2).

\section{Predator swimming speed and body size}

In both the monoculture and the mixed culture, the predator revealed two distinct swimming regimes (supplementary

Fig. S6a). This was further confirmed by a hierarchical cluster analysis of the trajectories (see Section S2.1). Hence, 
we classified predator individuals as either slow or fast, depending on whether it's swimming speed was less or greater than $1500 \mu \mathrm{m} \mathrm{s}^{-1}$, respectively.

We fitted the skew normal distributions separately to the swimming speed data of the fast and the slow predator (Figs. 1a,b). On average, slow-swimming and fast-swimming predator individuals swam respectively $6.3 \%$ and $16.6 \%$ slower in the mixed culture than in the monoculture (non-overlapping 95\% confidence intervals, see Fig. 1c and Table S3). This difference was independent from the length of the trajectories considered (Fig. S7). There was significantly less variation in swimming speed for the fast predator in monoculture when compared to the mixed culture, while no other differences in the standard deviation and the skewness of the swimming speed distributions where present (Figs. S6e,f).

The body size of the fast-swimming predator significantly increased by $17.7 \%$ in the mixed culture (non-overlapping 95\% confidence intervals, see Figs. $2 b$, f and Table S9). This was not the case for the slow predator, where the increase by $4.1 \%$ in body size in the mixed culture was not significantly different from 0 (overlapping $95 \%$ confidence intervals, see Figs. 2a,f). Further, the swimming speed of the slow predator increased by $43.7 \mu \mathrm{m} \mathrm{s}^{-1}(t$-value=7.04, $\mathrm{df}=266, p$ value $<0.001)$ for each $1000 \mu \mathrm{m}^{2}$ increase of its body size, with no difference between culture types $(t$-value $=0.87$, $\mathrm{df}=266, p$-value $=0.385$, see Fig. $2 \mathrm{~d}$ and Table S10). There was no significant relation between body size and swimming speed $(t$-value $=0.45, \mathrm{df}=1400, p$-value $=0.652)$ for the fast predator, while it was confirmed that it swam slower in mixed culture $(t$-value $=5.73, \mathrm{df}=1400, p$-value $<0.001)$.

\section{Prey swimming speed and body size}

The prey showed two swimming speed regimes also, as the distribution was bimodal (Fig. 1d). The proportion of prey individuals showing the fast movement behavior increased by $12.5 \%$ from $65.3 \%$ to $77.8 \%$ in the presence of the predator (non-overlapping 95\% confidence intervals in Fig. 1e and Table S4). We found this difference also for trajectories of only a certain length range (Fig. S10) and also when for the prey mixed culture we only considered videos in which the prey abundance was comparable to the prey abundances in the monoculture (Fig. S2 and Fig. S11). The mean slow swimming speed (i.e., $\mu_{1}$ ) was $7.8 \%$ greater in the monoculture compared to the mixed culture, the other parameters were not significantly different between the two cultures based on their $95 \%$ confidence intervals, (Figs. S9b,c). 
The prey body size was not significantly affected by the presence of the predator (overlapping $95 \%$ confidence intervals, see Figs. 2c,f and Table S9). For every increase in body size of $1000 \mu \mathrm{m}^{2}$ the prey swam 84.3 and $92.2 \mu \mathrm{m}$ $\mathrm{s}^{-1}$ faster $(t$-value $=36.46, \mathrm{df}=9707, p$-value $<0.001)$, respectively in mono- and the mixed culture, with a significant difference between the two slopes $(t$-value $=-2.64, \mathrm{df}=9707, p$-value=0.008, see Fig. 2e and Table S10).

\section{Predator displacements}

The predator displacements were smaller in the presence of prey (non-overlapping confidence intervals in Fig. 3a) and showed superdiffusion in all cases (slope $\gamma>1$ in Fig. 3b). The MSD values after one second were $27.0 \%$ and $12.5 \%$ smaller in mixed culture, respectively for the slow- and the fast-swimming predator (Table S5). After 9 seconds (the largest time-lag value considered for the MSD) these values were respectively $35.9 \%$ and $8.4 \%$ but with overlapping $95 \%$ confidence intervals in the case of the fast-swimming predator likely caused by the small number of trajectories that were 9 seconds long or longer.

Predator individuals also showed displacements that were dependent on the local number of neighbors (Fig. 4). Both the slow- and the fast-swimming predator made smaller displacements the more local prey neighbors they had, with the latter also making smaller movements when it had more predator neighbors in the monoculture (Fig. 4b). Quantitatively, with respect to its biggest displacement the predator made $2.3 \%(t$-value $=-2.70, \mathrm{df}=8946, p$ value $=0.007)$ and $3.7 \%(t$-value $=-3.28, \mathrm{df}=3918, p$-value $=0.001)$ smaller displacements for each unit increase in number of local prey neighbors, respectively for the fast- and the slow-swimming predator (Table S6). These patterns persisted in the sensitivity analysis of different neighbor detection ranges (Table S6). The analysis of predator swimming speed showed that the predator swam faster in the monoculture than in the mixed culture regardless of number of neighbors, suggesting that the presence or absence of prey had a larger impact on predator swimming speed than the number of neighbors (see supplementary Fig. S12).

\section{Prey grouping}

On average the grouping index was larger in the mixed culture replicates, but it also showed considerable variability in time (Fig. 5a). The mixed effects model revealed evidence for increased grouping in presence of the predator, with the prey grouping $7.8 \%$ more in the mixed culture at time $0(t$-value $=-4.54, \mathrm{df}=713, p$-value $<0.001$, see Fig. $5 \mathrm{~b}$ and 


\section{Discussion}

263 We found evidence of both non-consumptive effects (NCEs) of predators on prey, and of resource availability effects

Table S8). However, the 95\% confidence intervals overlapped in later parts of the videos, suggesting that the relation between predator presence and prey grouping cannot be clearly answered with this data. The alternative analysis based on intensity functions yielded comparable results (see Fig. S15 and Table S8). Further, no difference in prey grouping was found when only videos were considered in which the prey abundance was comparable between the mixed and the monoculture (Fig. S16).

(RAEs) of prey on predators. One NCE was the proportionally greater number of prey individuals swimming faster in the presence of predators. RAEs included the presence of prey causing predators to significantly decrease swimming speed and displacements, and to increase body size. Predator displacements were also dependent on local prey density. Together, these results suggest that the predator counteracts the prey scarcity by changing its behavior to search more quickly and in larger areas, in a likely attempt to increase the encounter rate with the prey, while the prey reacts to high predator densities by swimming faster and thus by possibly increasing the evasion rate upon encounter with the predator, though while also likely increasing encounter rates.

NCEs are a frequently studied component of predator-prey interactions (see for instance Lima 1998; Werner and Peacor 2003; Hermann and Landis 2017; MacLeod et al. 2018). The change in behavior of the average prey individual being more likely to exhibit the fast-swimming speed in the presence of the predator is confirmed by similar results (e.g. Broglio et al. 2001; Hammill et al. 2009) and represents evidence for flight behavior, as it was presumably made by the prey to increase its escape ability upon encounter with the predator (Sih 2011). Flight as an anti-predator behavior is a widespread phenomena (see for instance Krause and Godin 1996; Stankowich 2009; Harvey and Menden-Deuer 2012) and has also been reported for ciliates (Tamar 1979). As the predator was relatively abundant in the mixed cultures, it is further possible that the prey was in a constant state of flight behavior. This could potentially also explain the only weak evidence for increased prey grouping caused by predator presence, a behavior often observed in animals (e.g. tadpoles and cercopithecoid primates, see respectively Hill and Lee 1998; Spieler 2003). 
281 Contrasting this, a lot less is known about RAEs. The investigation of trait mediated interactions in predator-prey systems is most often centered around the prey and how it evades or escapes the predator (see for example Wahl and Stein 1988; Miller et al. 2014) and much less frequently on the predator and how it changes its search strategy at low resource availability (e.g. Munk 1995; Ronconi and Burger 2008). Rare evidence for an increased predator swimming speed in the absence of prey was reported by Kuefler et al. (2013), who similar to our results found that on average their studied rotifer species swam faster in absence of food but only when there were no conspecific individuals present. Assuming that increased movement rates and displacement lengths are likely to be associated with a better chance of encountering prey, the here reported increase in swimming speed and displacements represents evidence that the predator Didinium nasutum is capable of responding to increased hunger levels by not only covering more space but also by searching in it more quickly. On this note, larger predator displacements as a counter to the lack of food, either to search in larger areas or to move to other patches altogether, have been reported in the context of Lévy flights (a particular search strategy, see e.g. Humphries et al. 2010; Sims et al. 2012).

With the presence of both RAEs and NCEs confirmed in such a widely studied predator-prey pair, it becomes evident that neglecting these effects when the interaction strength between species is assessed is questionable. However, when the interaction strength is simplified to correspond to the functional response, such neglect is caused, i.e. it is assumed that prey intrinsic growth rate is independent from the predator density and that predator conversion efficiency and mortality rate are invariant to prey availability (Rall et al. 2010; Sentis et al. 2012; Kalinoski and DeLong 2016; Daugaard et al. 2019). Yet, it has long been known that prey growth does, in fact, depend on predator density (Preisser et al. 2005; Clinchy et al. 2013) while it has recently also been pointed out that the conversion efficiency and the mortality rate are associated with prey abundance (Minter et al. 2011; Li and Montagnes 2015). Crucially, the functional response approach only estimates the consumptive effects of predators on prey and can only partially and implicitly incorporate NCEs and RAEs. For example, while induced defensive strategies may cause a decrease in the predator feeding rates and are thus at least in part captured by the functional response parameters, this approach does neither incorporate the growth rate of the prey nor any (e.g. predator-mediated) changes in it. Ultimately, concrete

305 knowledge about predation effects other than predator feeding rates, such as changes in prey behavior in predator 
characteristics of the system (e.g. whether the predator exerts higher or lower predation pressure on the prey when the prey is only present in small numbers).

The estimated predator swimming speed revealed two clearly different movement rate regimes (i.e., the slow- and the fast-swimming predator). The only other study finding similar results did so in a heavily altered medium (in which the average swimming speed reached only $3 \%$ of the swimming speed in the unaltered medium, see Luckinbill 1973). It is possible that the more modern videography approach used in our study was the factor that allowed the detection of the two swimming speed regimes in a more natural setting that has been overlooked so far and that represents a gap in the understanding of D. nasutum.

A potential explanation for the presence of this bimodal swimming behavior could be that individuals swimming slower are the ones that recently went through reproduction, which would explain why there are proportionally many more fast individuals than slow ones (Table S1). Considering that the slow predator was generally also smaller than the fast predator in both the mixed and the monocultures (Fig. 2f), this further suggests that the slow predator recently went through cell division (i.e. reproduction). More evidence in favor of this is that only the body size of the fast predator decreased in the absence of prey (i.e. due to starvation), while the slow predator did not significantly change in size it is possible that only individuals of a certain size go through cell division. The analysis of the relation between morphology and speed also revealed that the swimming speed of both prey and slow predators increased with body size (Figs. 2d,e). These results suggest that increasing the body size leads to higher swimming speeds, but with diminishing returns as the movement of the fast predator was independent from body size.

While the described NCEs and RAEs for this species pair are likely to influence the prey-predator interaction strength and thus the population dynamics of the two species, we do not quantify this here. This represents an interesting research question for further studies, that could for instance extend the work of Griffiths et al. (2018) in which a traitdemography feedback with predator-dependent prey body size was modeled.

Jointly considered, the results show that both the predator Didinium nasutum and the prey Paramecium caudatum are able to behaviorally and morphologically (only Didinium nasutum) react to changes in abundance of their trophic opponent. This is expected, as to maximize their fitness organisms must find a balance between foraging and avoiding being eaten by a predator and therefore the behavior of both the prey and the predator depends on the environment and 


\section{$342 \quad$ Funding}

The research was supported by Swiss National Science Foundation Project 310030_188431

\section{Author contributions}

345 UD, RF and OLP conceived the ideas and designed the methodology. UD collected and analyzed the data and led the 346 writing of the manuscript. All authors contributed critically to the drafts and gave final approval for publication.

\section{Ethics declarations}

348 Competing interests: The authors declare that they have no competing interests.

349 Ethical approval: This is a study with protists and as such no ethical approval is required.

\section{Data availability}

351 Data available from the Zenodo repository: https://doi.org/10.5281/zenodo.5676309 (Daugaard et al. 2021) 


\section{References}

353 Abrams PA, Ginzburg LR (2000) The nature of predation: prey dependent, ratio dependent or neither? Trends Ecol Evol 15:337-341. https://doi.org/10.1016/S0169-5347(00)01908-X

Altermatt F, Fronhofer EA, Garnier A, et al (2015) Big answers from small worlds: a user's guide for protist microcosms as a model system in ecology and evolution. Methods Ecol Evol 6:218-231. https://doi.org/10.1111/2041-210X.12312

Anholt BR, Werner EE (1995) Interaction between food availability and predation mortality mediated by adaptive behavior. Ecology 76:2230-2234. https://doi.org/10.2307/1941696

Ariel G, Rabani A, Benisty S, et al (2015) Swarming bacteria migrate by Lévy Walk. Nat Commun 6:8396. https://doi.org/10.1038/ncomms9396

Berlow EL, Neutel A-M, Cohen JE, et al (2004) Interaction strengths in food webs: issues and opportunities. J Anim Ecol 73:585-598. https://doi.org/10.1111/j.0021-8790.2004.00833.X

Bolker B, Team RDC (2017) bbmle: Tools for General Maximum Likelihood Estimation

Bozer S, Traugott M, Stamp N (1996) Combined effects of allelochemical-fed and scarce prey on the generalist insect predator Podisusmaculiventris. Ecol Entomol 21:328-334. https://doi.org/10.1046/j.1365-2311.1996.00011.x

Broglio E, Johansson M, Jonsson PR (2001) Trophic interaction between copepods and ciliates: effects of prey swimming behavior on predation risk. Mar Ecol Prog Ser 220:179-186. https://doi.org/10.3354/meps220179

Chesson P (2000) Mechanisms of maintenance of species diversity. Annu Rev Ecol Syst 31:343-366. https://doi.org/10.1146/annurev.ecolsys.31.1.343

Clinchy M, Sheriff MJ, Zanette LY (2013) Predator-induced stress and the ecology of fear. Funct Ecol 27:56-65. https://doi.org/10.1111/1365-2435.12007 
Daugaard U, Furrer R, Petchey OL (2021) Data from: Prey speed up, predators slow down: non- consumptive effects on movement behavior of a ciliate predator-prey pair. Version v1.0. Zenodo. URL https://doi.org/10.5281/zenodo.5676309

Daugaard U, Petchey OL, Pennekamp F (2019) Warming can destabilize predator-prey interactions by shifting the functional response from Type III to Type II. J Anim Ecol 88:1575-1586. https://doi.org/10.1111/13652656.13053

Dexter JP, Prabakaran S, Gunawardena J (2019) A complex hierarchy of avoidance behaviors in a single-cell eukaryote. Curr Biol 29:4323-4329. https://doi.org/10.1016/j.cub.2019.10.059

Fernández C, Steel MF (1998) On Bayesian modeling of fat tails and skewness. J Am Stat Assoc 93:359-371. https://doi.org/10.1080/01621459.1998.10474117

Fill A, Long EY, Finke DL (2012) Non-consumptive effects of a natural enemy on a non-prey herbivore population. Ecol Entomol 37:43-50. https://doi.org/10.1111/j.1365-2311.2011.01333.x

Griffiths JI, Petchey OL, Pennekamp F, Childs DZ (2018) Linking intraspecific trait variation to community abundance dynamics improves ecological predictability by revealing a growth-defence trade-off. Funct Ecol 32:496-508. https://doi.org/10.1111/1365-2435.12997

Gurarie E, Grünbaum D, Nishizaki MT (2011) Estimating 3D Movements from 2D Observations Using a Continuous Model of Helical Swimming. Bull Math Biol 73:1358-1377. https://doi.org/10.1007/s11538-010-9575-7

Hammill E, Kratina P, Anholt BR (2009) Non-lethal presence of predators modifies morphology and movement rates in Euplotes. Hydrobiologia 621:183-189. https://doi.org/10.1007/s10750-008-9644-1

Hammill E, Petchey OL, Anholt BR (2010) Predator functional response changed by induced defenses in prey. Am Nat 176:723-731. https://doi.org/10.1086/657040 
Harvey EL, Menden-Deuer S (2012) Predator-induced fleeing behaviors in phytoplankton: a new mechanism for harmful algal bloom formation? PloS One 7:. https://doi.org/10.1371/journal.pone.0046438

Hermann SL, Landis DA (2017) Scaling up our understanding of non-consumptive effects in insect systems. Curr Opin Insect Sci 20:54-60. https://doi.org/10.1016/j.cois.2017.03.010

Hewett SW (1980) Prey-Dependent Cell Size In A Protozoan Predator; PREY SIZE and Didinium SIZE. J Protozool 27:311-313. https://doi.org/10.1111/j.1550-7408.1980.tb04263.x

Hewett SW (1988) Predation by Didinium nasutum: effects of predator and prey size. Ecology 69:135-145. https://doi.org/10.2307/1943168

Hill R, Lee P (1998) Predation risk as an influence on group size in cercopithecoid primates: implications for social structure. J Zool 245:447-456. https://doi.org/10.1111/j.1469-7998.1998.tb00119.x

Humphries NE, Queiroz N, Dyer JRM, et al (2010) Environmental context explains Lévy and Brownian movement patterns of marine predators. Nature 465:1066-1069. https://doi.org/10.1038/nature09116

Janczura J, Weron A (2015) Ergodicity testing for anomalous diffusion: Small sample statistics. J Chem Phys 142:144103. https://doi.org/10.1063/1.4916912

Jennings HS (1902) Studies on reactions to stimuli in unicellular organisms. IX. — On the behavior of fixed infusoria (Stentor and Vorticella), with special reference to the modifiability of protozoan reactions. Am J Physiol-Leg Content 8:23-60. https://doi.org/10.1152/ajplegacy.1902.8.1.23

Kadoya T, Gellner G, McCann KS (2018) Potential oscillators and keystone modules in food webs. Ecol Lett 21:1330-1340. https://doi.org/10.1111/ele.13099

Kahl A (1930) Urtiere oder Protozoa. I. Wimpertiere oder Ciliate (Infusoria). 1. Allgemeiner Teil und Prostomata. Tierwelt Angrenzenden Meeresteile 18:1-180 
415 Kalinoski RM, DeLong JP (2016) Beyond body mass: how prey traits improve predictions of functional response parameters. Oecologia 180:543-550. https://doi.org/10.1007/s00442-015-3487-z

Krause J, Godin J-GJ (1996) Influence of prey foraging posture on flight behavior and predation risk: predators take advantage of unwary prey. Behav Ecol 7:264-271. https://doi.org/10.1093/beheco/7.3.264 Ecol 27:323-328. https://doi.org/10.1111/1365-2435.12065

Kusch J (1993) Behavioural and morphological changes in ciliates induced by the predator Amoeba proteus. Oecologia 96:354-359. https://doi.org/10.1007/bf00317505 https://doi.org/10.2307/1313225 nasutum. Ecology 54:1320-1327. https://doi.org/10.2307/1934194 https://doi.org/10.2307/1940365 non-consumptive predation risk. Oikos 127:375-380. https://doi.org/10.1111/oik.04890 
Méndez V, Campos D, Bartumeus F (2014) Anomalous Diffusion and Continuous-Time Random Walks. In: Méndez V, Campos D, Bartumeus F (eds) Stochastic Foundations in Movement Ecology: Anomalous Diffusion, Front Propagation and Random Searches. Springer, Berlin, Heidelberg, pp 113-148

Miller JR, Ament JM, Schmitz OJ (2014) Fear on the move: predator hunting mode predicts variation in prey mortality and plasticity in prey spatial response. J Anim Ecol 83:214-222. https://doi.org/10.1111/13652656.12111

Mills LS, Soulé ME, Doak DF (1993) The keystone-species concept in ecology and conservation. BioScience 43:219224. https://doi.org/10.2307/1312122

Minter EJA, Fenton A, Cooper J, Montagnes DJS (2011) Prey-Dependent Mortality Rate: A Critical Parameter in Microbial Models. Microb Ecol 62:155-161. https://doi.org/10.1007/s00248-011-9836-5

Munk P (1995) Foraging behaviour of larval cod (Gadus morhua) influenced by prey density and hunger. Mar Biol $122: 205-212$

O’brien WJ, Browman HI, Evans BI (1990) Search strategies of foraging animals. Am Sci 78:152-160

O’Gorman EJ, Emmerson MC (2009) Perturbations to trophic interactions and the stability of complex food webs. Proc Natl Acad Sci 106:13393-13398. https://doi.org/10.1073/pnas.0903682106

Paine RT (1969) A note on trophic complexity and community stability. Am Nat 103:91-93. https://doi.org/10.1086/282586

Peckarsky BL, Abrams PA, Bolnick DI, et al (2008) Revisiting the classics: considering nonconsumptive effects in textbook examples of predator-prey interactions. Ecology 89:2416-2425. https://doi.org/10.1890/07-1131.1

Pennekamp F, Schtickzelle N, Petchey OL (2015) BEMOVI, software for extracting behavior and morphology from videos, illustrated with analyses of microbes. Ecol Evol 5:2584-2595. https://doi.org/10.1002/ece3.1529 
Preisser EL, Bolnick DI (2008) The Many Faces of Fear: Comparing the Pathways and Impacts of Nonconsumptive Predator Effects on Prey Populations. PLOS ONE 3:e2465. https://doi.org/10.1371/journal.pone.0002465

Preisser EL, Bolnick DI, Benard MF (2005) Scared to death? The effects of intimidation and consumption in predator-prey interactions. Ecology 86:501-509. https://doi.org/10.1890/04-0719

Rall BC, Vucic-Pestic O, Ehnes RB, Emmerson M, Brose U (2010) Temperature, predator-prey interaction strength and population stability. Glob Change Biol 16:2145-2157. https://doi.org/10.1111/j.1365-2486.2009.02124.x

Ronconi RA, Burger AE (2008) Limited foraging flexibility: increased foraging effort by a marine predator does not buffer against scarce prey. Mar Ecol Prog Ser 366:245-258. https://doi.org/10.3354/meps07529

Salt GW (1979) Density, starvation, and swimming rate in Didinium populations. Am Nat 113:135-143. https://doi.org/10.1086/283369

Schmitz O (2017) Predator and prey functional traits: understanding the adaptive machinery driving predator-prey interactions. F1000Research 6:. https://doi.org/10.12688/f1000research.11813.1

Sentis A, Hemptinne J-L, Brodeur J (2012) Using functional response modeling to investigate the effect of temperature on predator feeding rate and energetic efficiency. Oecologia 169:1117-1125. https://doi.org/10.1007/s00442-012-2255-6

Sheriff MJ, Peacor SD, Hawlena D, Thaker M (2020) Non-consumptive predator effects on prey population size: A dearth of evidence. J Anim Ecol 89:1302-1316. https://doi.org/10.1111/1365-2656.13213

Sih A (2011) Foraging Theory. In: Scheiner SM, Willig MR (eds) The theory of ecology. University of Chicago Press, pp 65-92

Sih A, Christensen B (2001) Optimal diet theory: when does it work, and when and why does it fail? Anim Behav 61:379-390. https://doi.org/10.1006/anbe.2000.1592 
Sims DW, Humphries NE, Bradford RW, Bruce BD (2012) Lévy flight and Brownian search patterns of a freeranging predator reflect different prey field characteristics. J Anim Ecol 81:432-442. https://doi.org/10.1111/j.1365-2656.2011.01914.x

Spieler M (2003) Risk of predation affects aggregation size: a study with tadpoles of Phrynomantis microps (Anura: Microhylidae). Anim Behav 65:179-184. https://doi.org/10.1006/anbe.2002.2030

Stankowich T (2009) When predators become prey: flight decisions in jumping spiders. Behav Ecol 20:318-327. https://doi.org/10.1093/beheco/arp004

Tamar H (1979) The movements of jumping ciliates. Arch Für Protistenkd 122:290-327. https://doi.org/10.1016/S0003-9365(79)80040-8

Van Buskirk J, Yurewicz KL (1998) Effects of Predators on Prey Growth Rate: Relative Contributions of Thinning and Reduced Activity. Oikos 82:20-28. https://doi.org/10.2307/3546913

van Veen FJF, van Holland PD, Godfray HCJ (2005) Stable Coexistence in Insect Communities Due to Density- and Trait-Mediated Indirect Effects. Ecology 86:3182-3189. https://doi.org/10.1890/04-1590

Wahl DH, Stein RA (1988) Selective predation by three esocids: the role of prey behavior and morphology. Trans Am Fish Soc 117:142-151. https://doi.org/10.1577/1548-8659(1988)117<0142:SPBTET>2.3.CO;2

Wajnberg E, Bernhard P, Hamelin F, Boivin G (2006) Optimal patch time allocation for time-limited foragers. Behav Ecol Sociobiol 60:1-10. https://doi.org/10.1007/s00265-005-0131-7

Werner EE, Peacor SD (2003) A Review of Trait-Mediated Indirect Interactions in Ecological Communities. Ecology 84:1083-1100. https://doi.org/10.1890/0012-9658(2003)084[1083:arotii]2.0.co;2

Wichterman R (2012) The biology of Paramecium. Springer Science \& Business Media

Wu C-H, Dahms H-U, Buskey EJ, Strickler JR, Hwang J-S (2010) Behavioral interactions of the copepod Temora turbinata with potential ciliate prey. Zool Stud 49:157-168 
bioRxiv preprint doi: https://doi.org/10.1101/2021.11.15.468607; this version posted November 16, 2021. The copyright holder for this preprint (which was not certified by peer review) is the author/funder. All rights reserved. No reuse allowed without permission.

500 Wuertz D, Setz T, Chalabi Y, Boudt C, Chausse P, Miklovac M (2019) fGarch: Rmetrics - Autoregressive 501 Conditional Heteroskedastic Modelling

502 Yodzis P (1994) Predator-Prey Theory and Management of Multispecies Fisheries. Ecol Appl 4:51-58.

503 https://doi.org/10.2307/1942114 


\section{Figures}

a

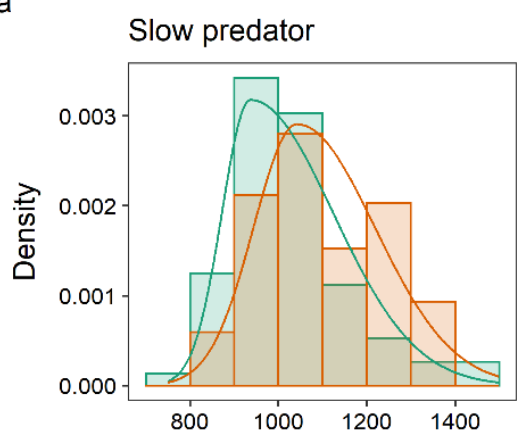

Swimming speed $[\mu \mathrm{m} / \mathrm{s}]$

d

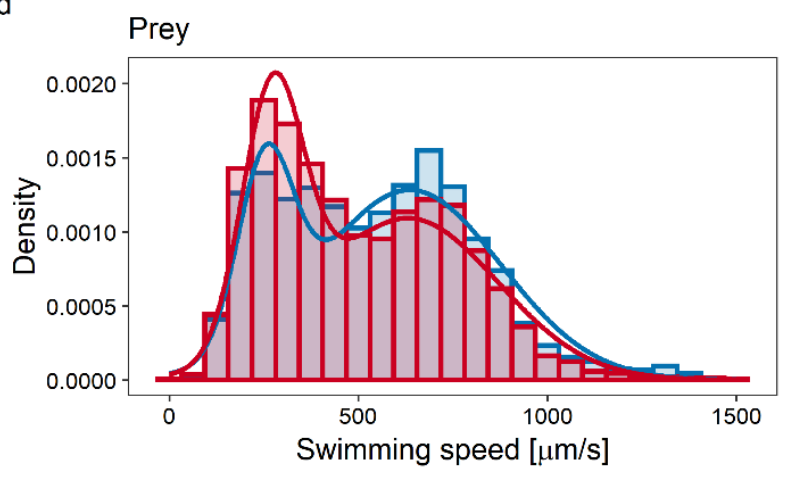

b

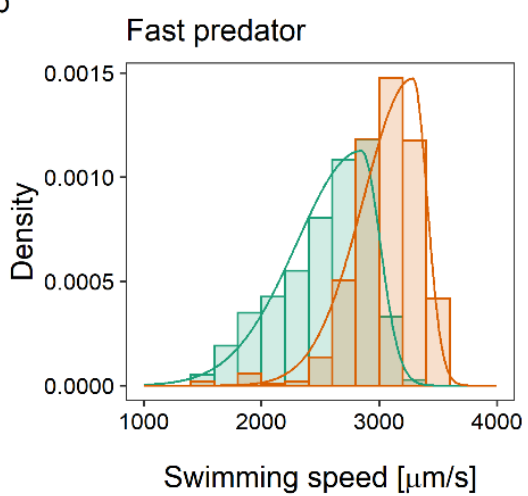

C

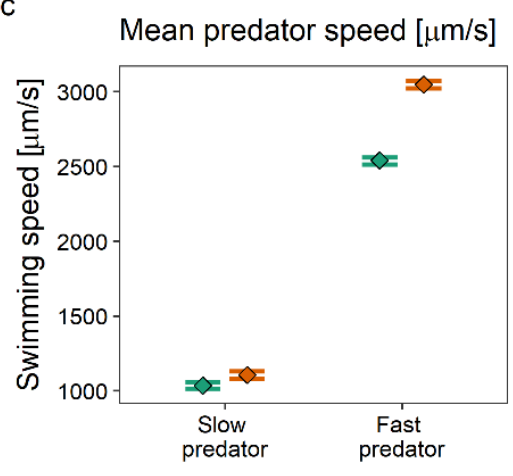

e

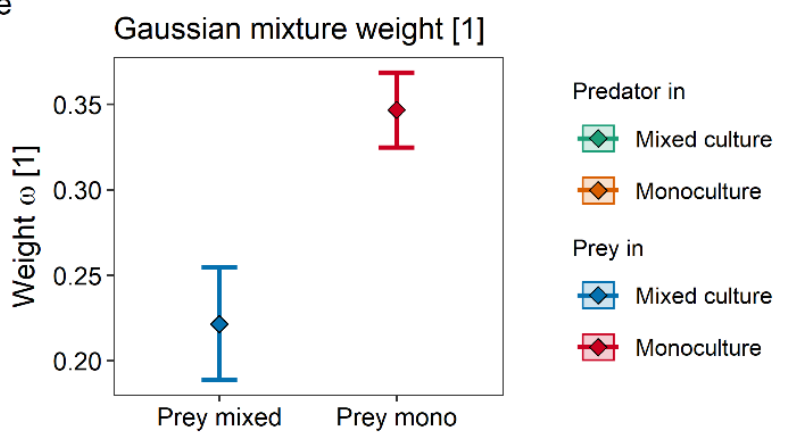

505

Fig. 1 a - b Empirical and fitted skew normal densities for the swimming speed of the predator in the two different cultures, respectively for the slow (a) and the fast (b) individuals. c Estimated mean swimming speed of the slow and the fast predator in mixed and monoculture. $\mathbf{d}$ Empirical and fitted Gaussian mixture densities for the swimming speed of the prey in the two different cultures. e Estimated Gaussian mixture weights for the swimming speed distribution of the prey in mixed and monoculture. In $\mathbf{c}$ and $\mathbf{e}$ the error bars indicate $95 \%$ confidence intervals 


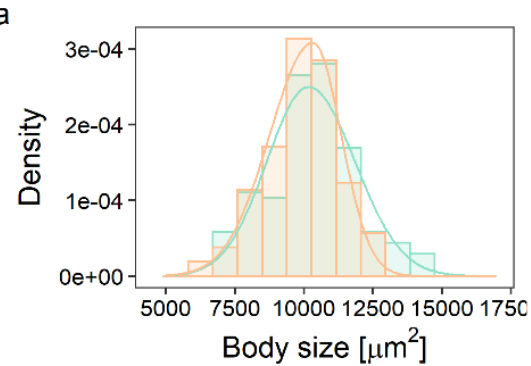

b

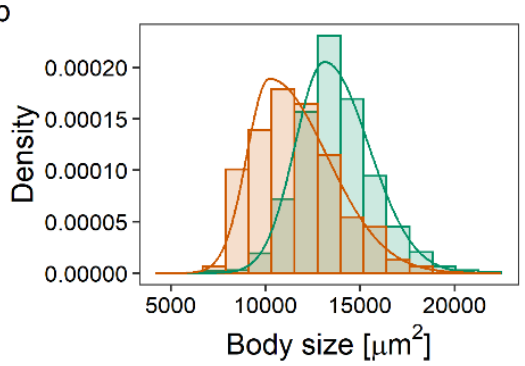

C

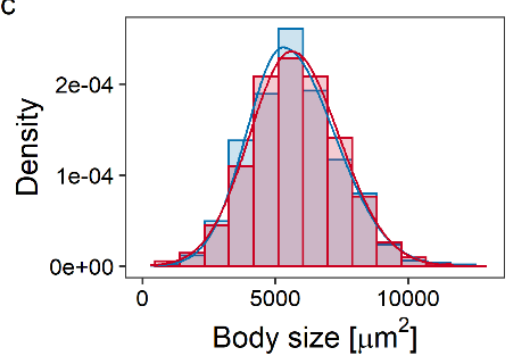

d
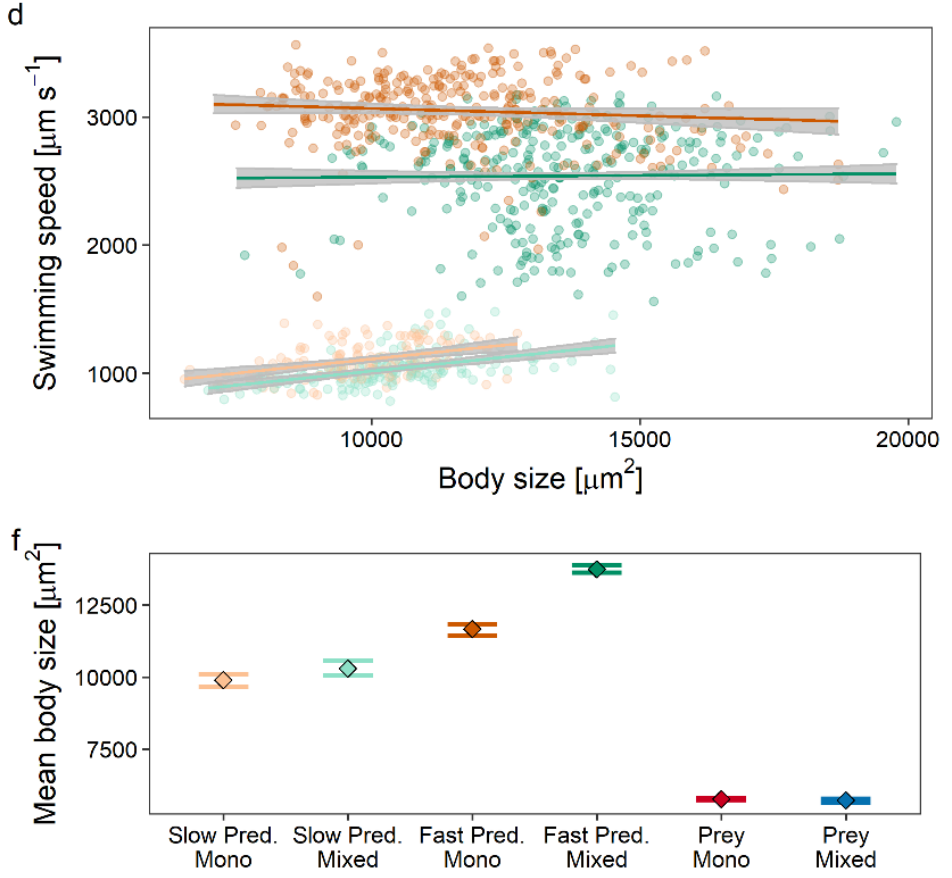

e

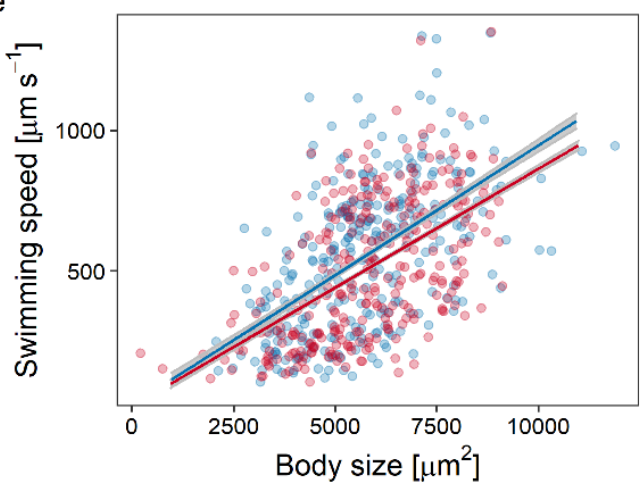

Slow predator in monoculture Slow predator in mixed culture Fast predator in monoculture Fast predator in mixed culture Prey in monoculture

Prey in mixed culture

512 Fig. 2 a - c Empirical and fitted skew normal densities for the body size of the two species in the two different cultures,

513 respectively for the slow predator (a), the fast predator (b) and the prey (c). $\mathbf{d}-\mathbf{e}$ The relation between species body

514 size and swimming speed in the two types of culture for the slow and the fast predator (d) and the prey (e). To avoid 515 over-plotting, we randomly selected 300 data points of each group to be displayed in a scatter-plot. Solid lines indicate regression fits and the shaded areas represent the pointwise $95 \%$ confidence intervals. f Estimated mean body sizes of the prey and the predator in presence and absence of each other. The error bars indicate $95 \%$ confidence intervals 
a

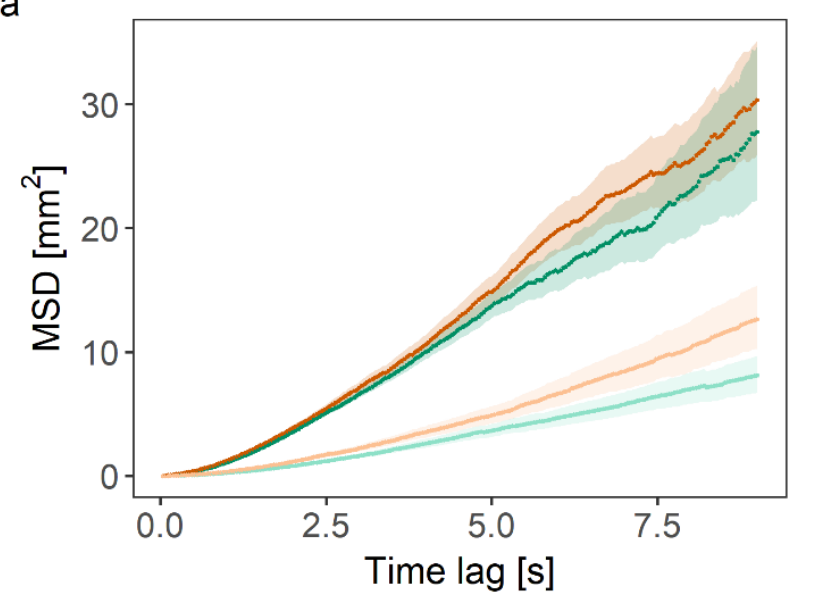

Fast predator in monoculture

- Fast predator in mixed culture b

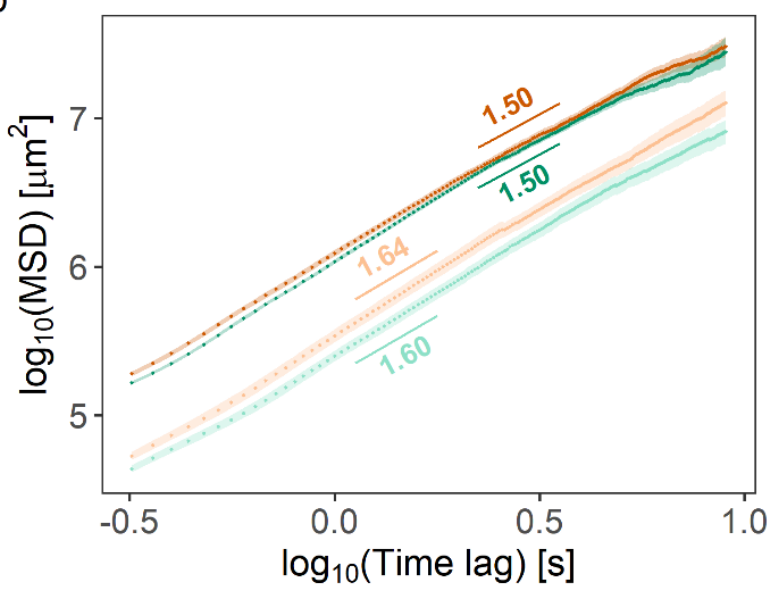

Slow predator in monoculture Slow predator in mixed culture

519 Fig. 3 Mean squared displacement (MSD) of the predator as a function of time, for both the predator monoculture and

520 the mixed culture, as well as for both the fast and the slow predator. Bootstrapped pointwise $95 \%$ confidence intervals

521 are included (shaded areas). a On normal axes. b On log10-transformed axes with corresponding slope estimates

522 displayed 
a
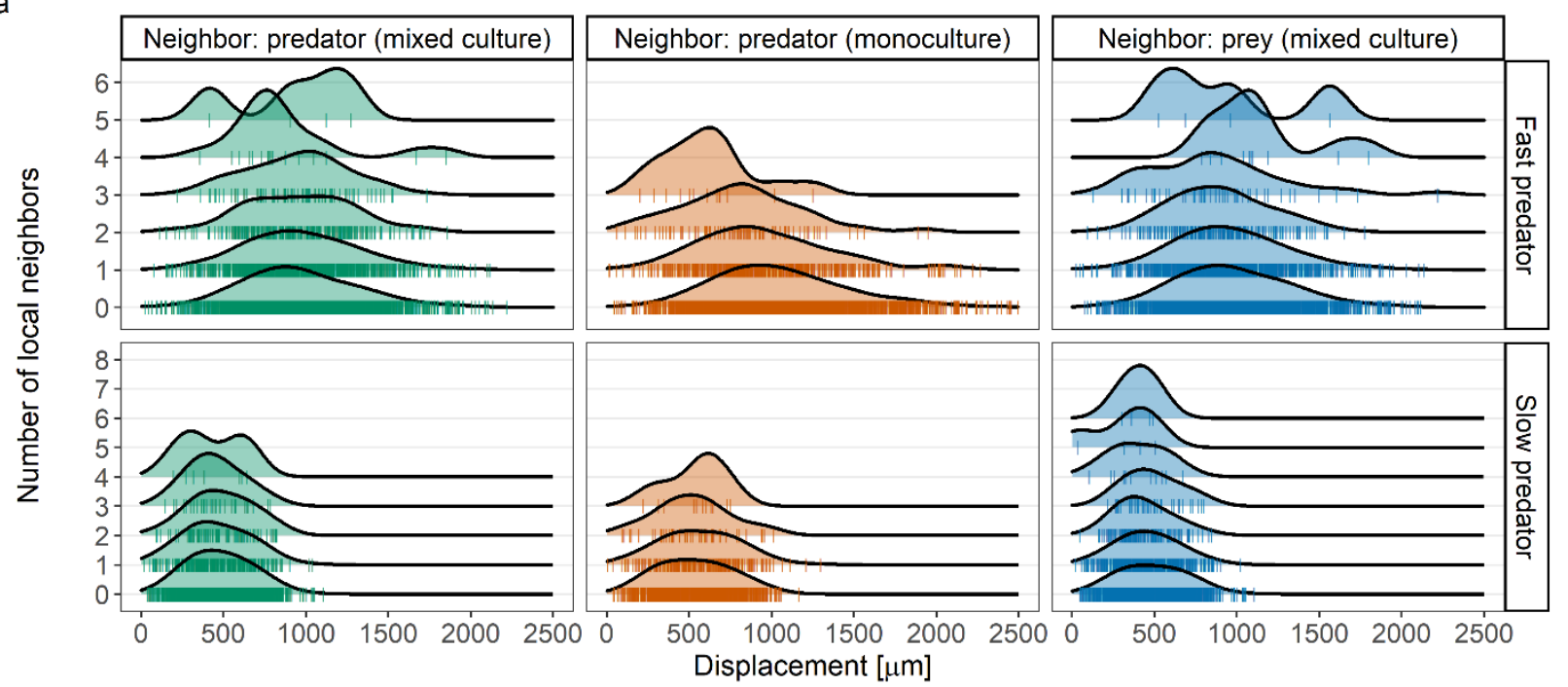

b

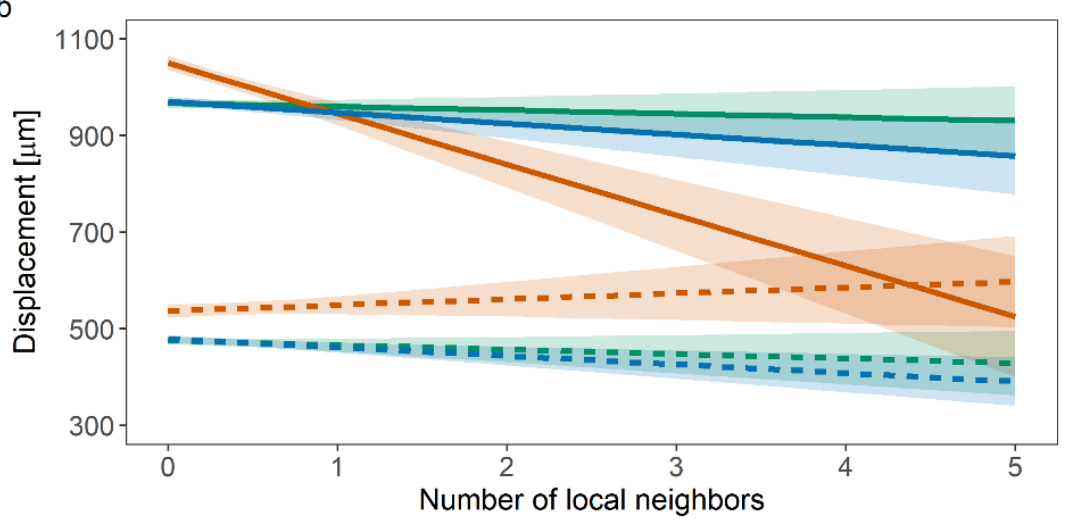

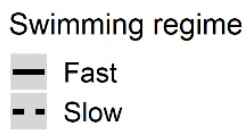

Neighbor type

- Predator (mixed culture)

- Predator (monoculture)

- Prey (mixed culture)

524 Fig. 4 Predator displacement per second as a function of the local number of neighbors at the start of the displacement.

a Ridge-line plots of the data including rug plots. The top row shows the fast-swimming predator, the bottom row the slow-swimming predator. The columns display the data for when the neighbors were predator individuals (columns

527 one and two, respectively for the mixed and the monocultures) and prey individuals (third column). b Results of the

528 linear regression (including $95 \%$ pointwise confidence intervals). The number of prey neighbors was set to zero if the

529 effect of predator neighbors was considered and vice versa. Solid lines represent the fast-swimming predator, dashed

530 lines the slow-swimming predator 

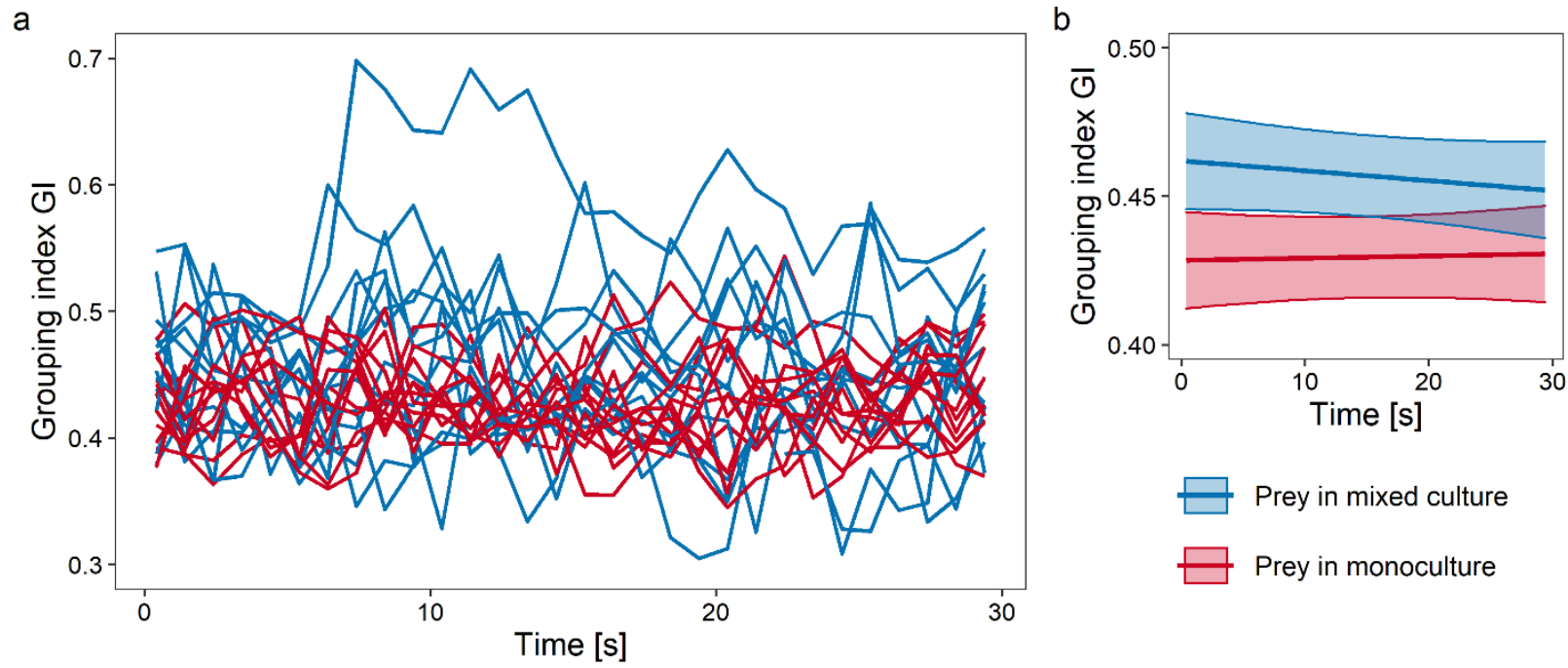

532 Fig. 5 Prey grouping factor as a function of culture type and time. a The calculated data, grouped by videos (lines).

533 b Results of the mixed effects models fitted. The pointwise $95 \%$ confidence intervals are based on fixed effects only 534 (shaded area) 


\section{Tables}

536 Table 1 Variables, their units, how they were calculated and their use (response or explanatory variable)

\begin{tabular}{|c|c|c|c|}
\hline Variable & Unit & Calculation & Used as \\
\hline $\begin{array}{l}\text { Predator swimming } \\
\text { speed }\end{array}$ & $\mu \mathrm{m} / \mathrm{s}$ & $\begin{array}{l}\text { Per trajectory: 60th percentile of recorded local swimming speed } \\
\text { maxima within trajectories. Across trajectories: skew normal } \\
\text { distribution fit to trajectory swimming speed distribution. }\end{array}$ & Response variable \\
\hline $\begin{array}{l}\text { Prey swimming } \\
\text { speed }\end{array}$ & $\mu \mathrm{m} / \mathrm{s}$ & $\begin{array}{l}\text { Per trajectory: median of recorded swimming speeds within the two- } \\
\text { dimensional parts of trajectories. Across trajectories: mixture of two } \\
\text { normal distributions fit to trajectory swimming speed distribution. }\end{array}$ & Response variable \\
\hline $\begin{array}{l}\text { Predator body } \\
\text { size }\end{array}$ & $\mu \mathrm{m} 2$ & $\begin{array}{l}\text { Per trajectory: mean of recorded body sizes. Across trajectories: } \\
\text { skew normal distribution fit to trajectory body size distribution. }\end{array}$ & $\begin{array}{l}\text { Response and } \\
\text { explanatory variable }\end{array}$ \\
\hline Prey body size & $\mu \mathrm{m} 2$ & $\begin{array}{l}\text { Per trajectory: mean of recorded body sizes recoded within the two } \\
\text { dimensional parts of trajectories. Across trajectories: skew } \\
\text { normal distribution fit to trajectory body size distribution. }\end{array}$ & $\begin{array}{l}\text { Response and } \\
\text { explanatory variable }\end{array}$ \\
\hline $\begin{array}{l}\text { MSD: Predator } \\
\text { displacements }\end{array}$ & $\mu \mathrm{m} 2$ & $\begin{array}{l}\text { The squared displacements of predators averaged over time (i.e. } \\
\text { within trajectories) and over the ensemble (i.e. across trajectories). }\end{array}$ & Response variable \\
\hline $\begin{array}{l}\text { Local prey and } \\
\text { predator } \\
\text { abundance }\end{array}$ & $\begin{array}{l}\text { cells/ } \\
\text { area }\end{array}$ & $\begin{array}{l}\text { For each individual, the local number of prey and predator was } \\
\text { determined as the number of respective individuals within a detection } \\
\text { radius of } 1500 \mu \mathrm{m} .\end{array}$ & Response variable \\
\hline Grouping Index (GI) & 1 & $\begin{array}{l}\text { One minus the empirical mean nearest neighbor divided by the } \\
\text { theoretical uniform neighbor distance. The resulting index can have } \\
\text { values between } 0 \text { (no grouping) and } 1 \text { (complete grouping) }\end{array}$ & Response variable \\
\hline
\end{tabular}


$a$

Slow predator

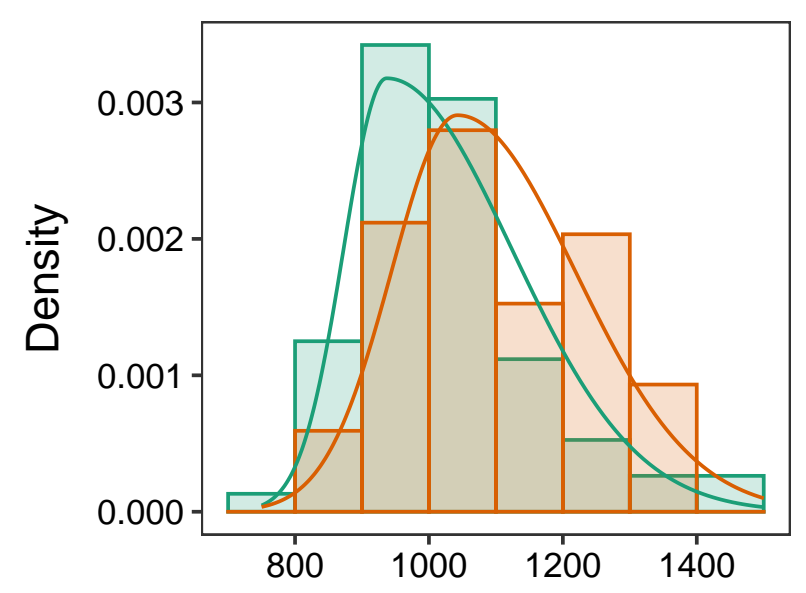

Swimming speed $[\mu \mathrm{m} / \mathrm{s}]$

d

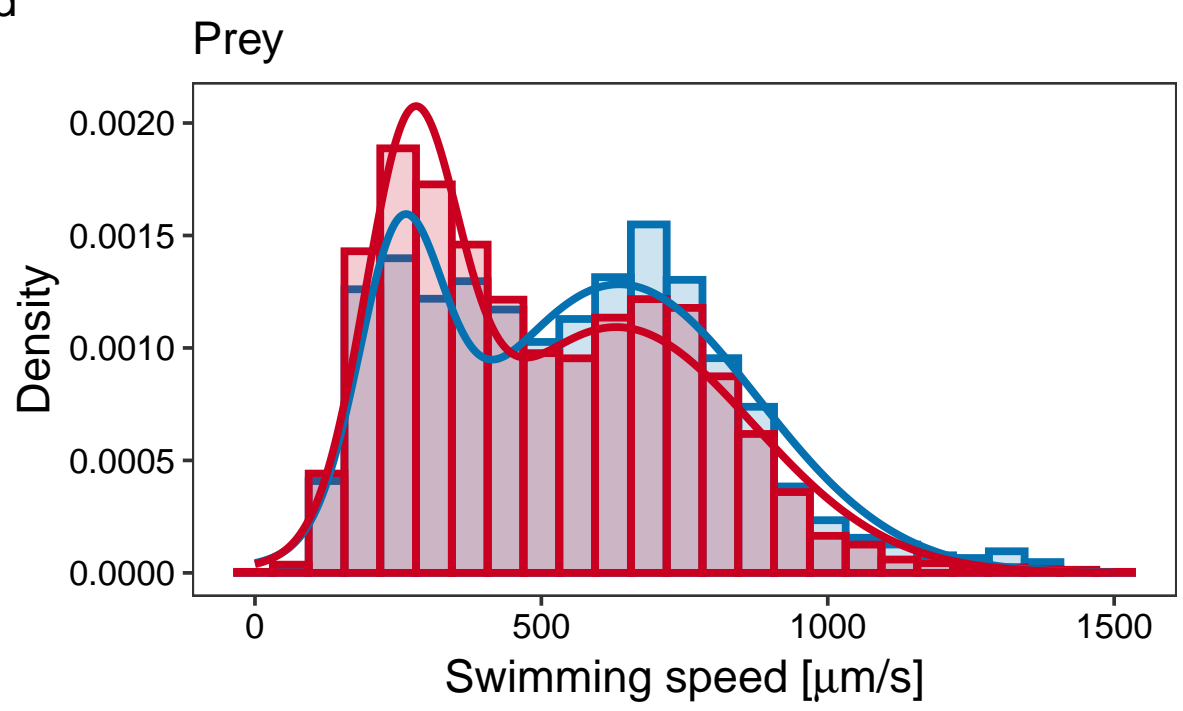

C
Fast predator

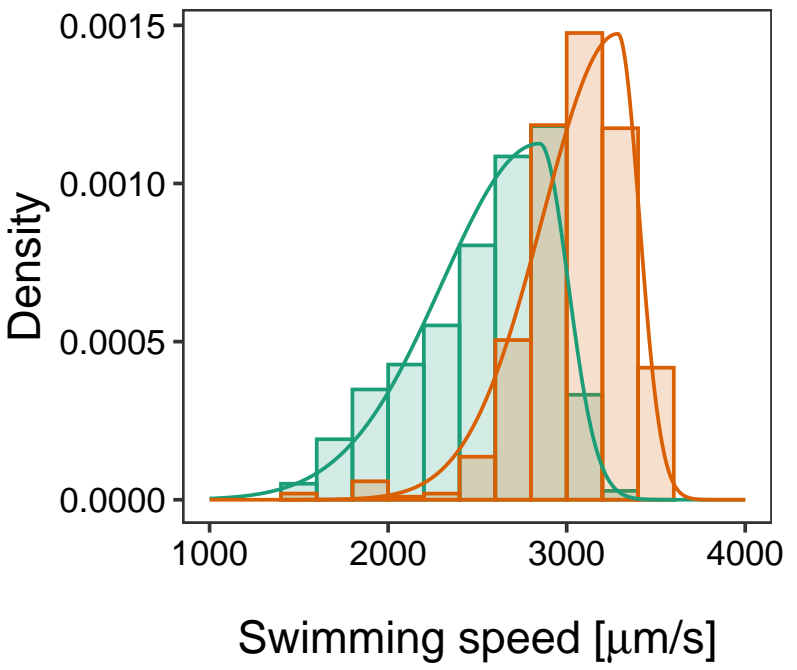

Mean predator speed $[\mu \mathrm{m} / \mathrm{s}]$

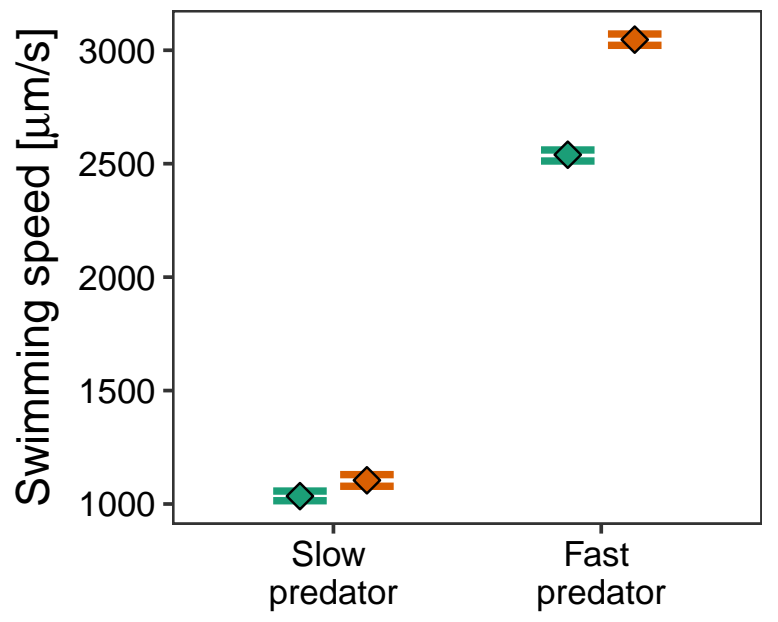

e

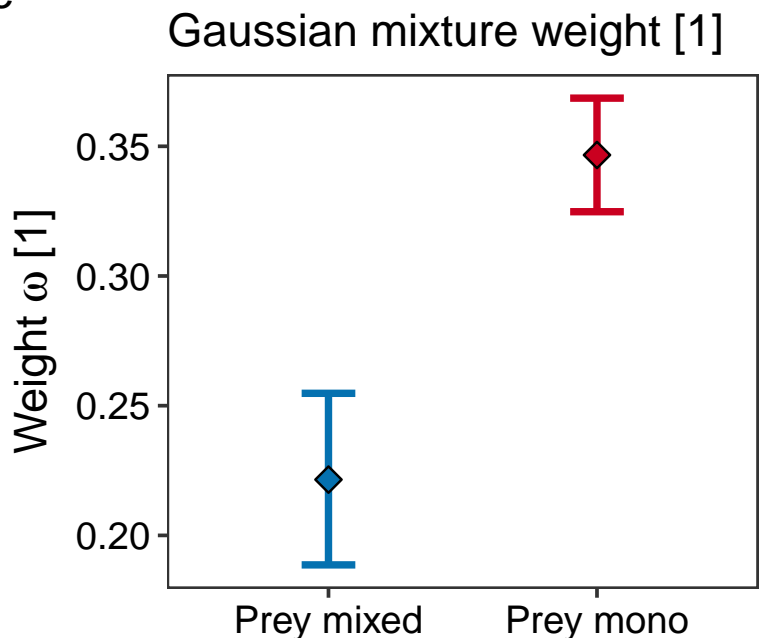

Predator in

$\nabla$ Mixed culture

$\nabla$ Monoculture

Prey in

$\nabla$ Mixed culture

$\nabla$ Monoculture 

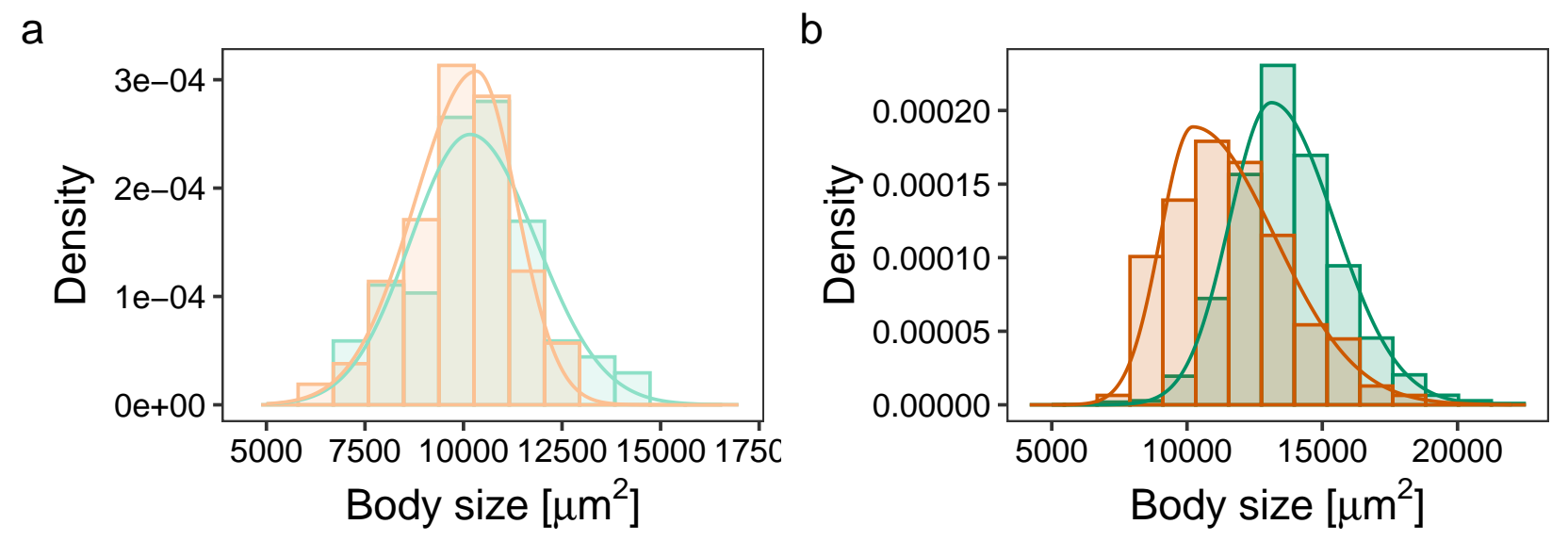

d
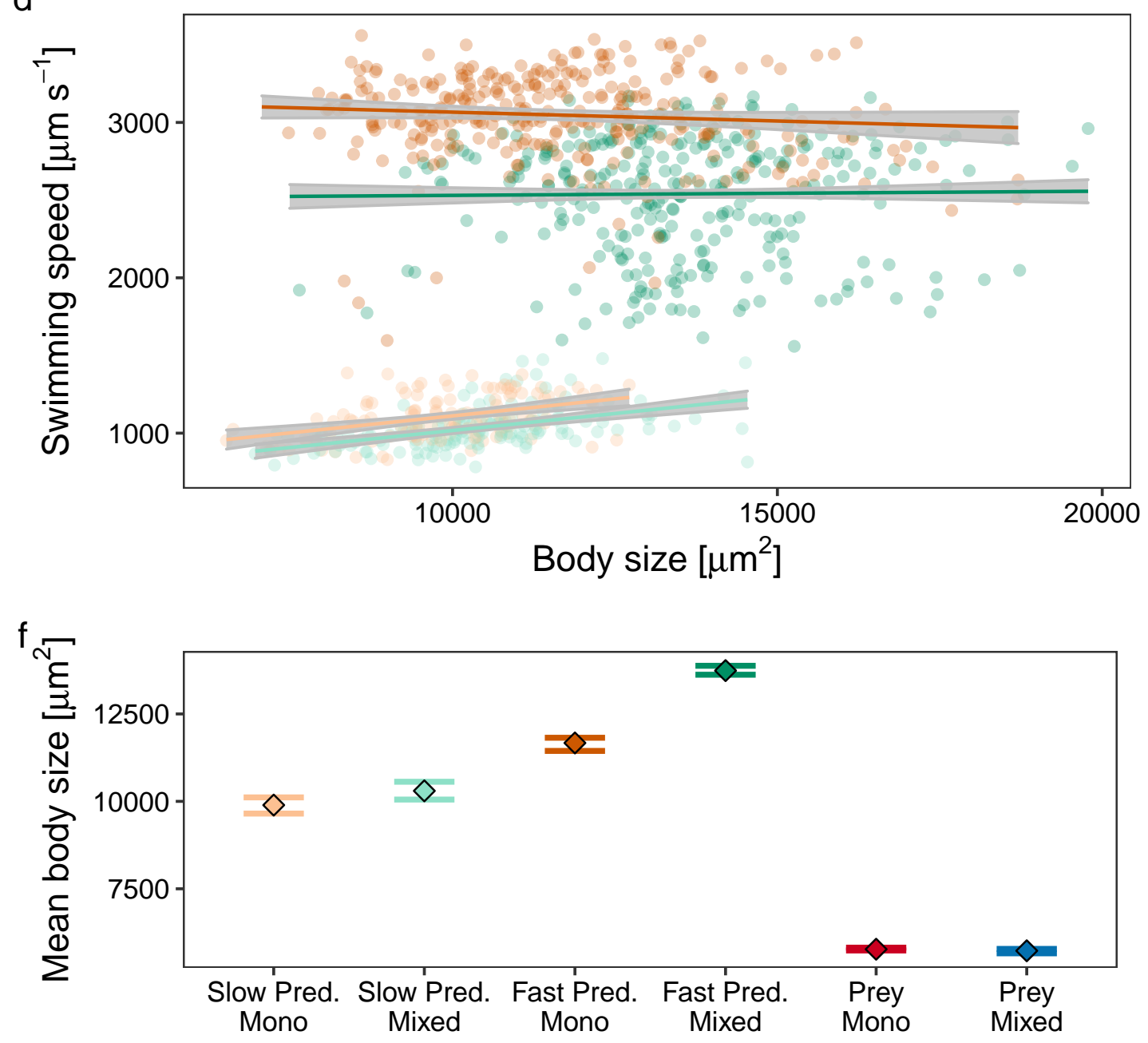

C

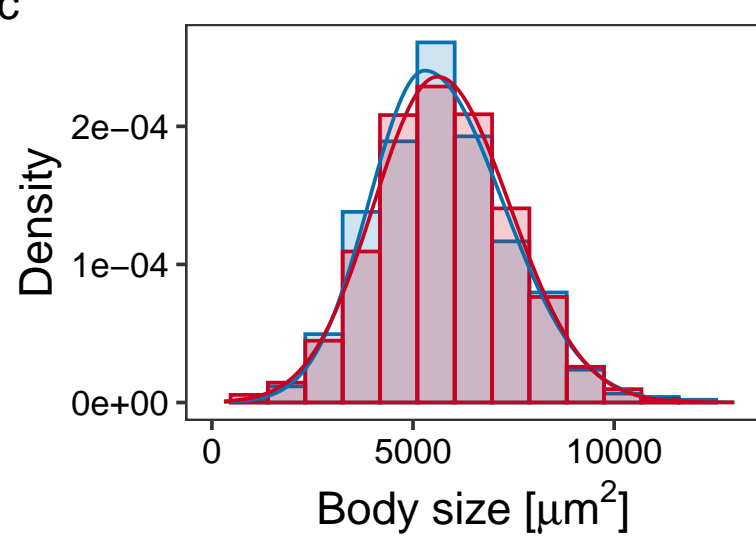

e

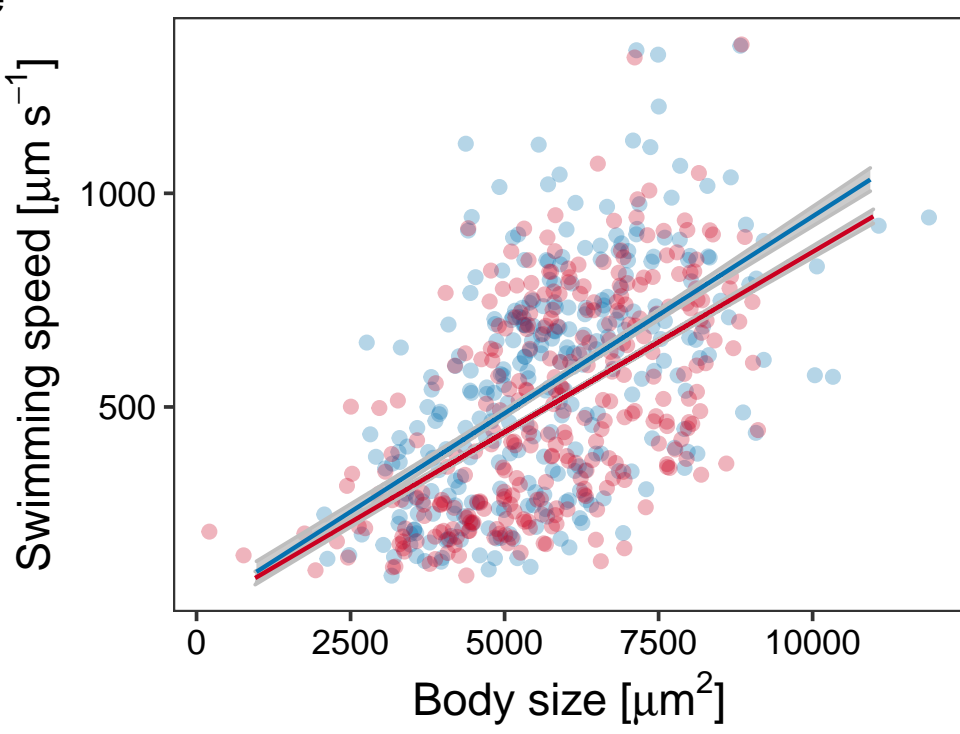

Slow predator in monoculture

Slow predator in mixed culture

Fast predator in monoculture

Fast predator in mixed culture

Prey in monoculture

Prey in mixed culture 
$a$

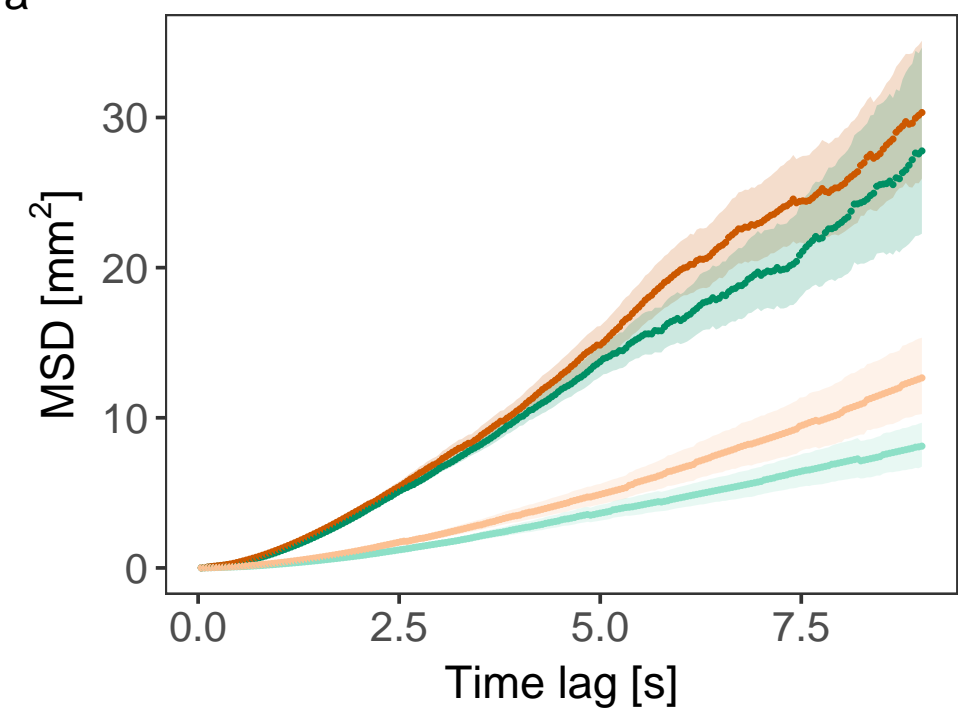

b

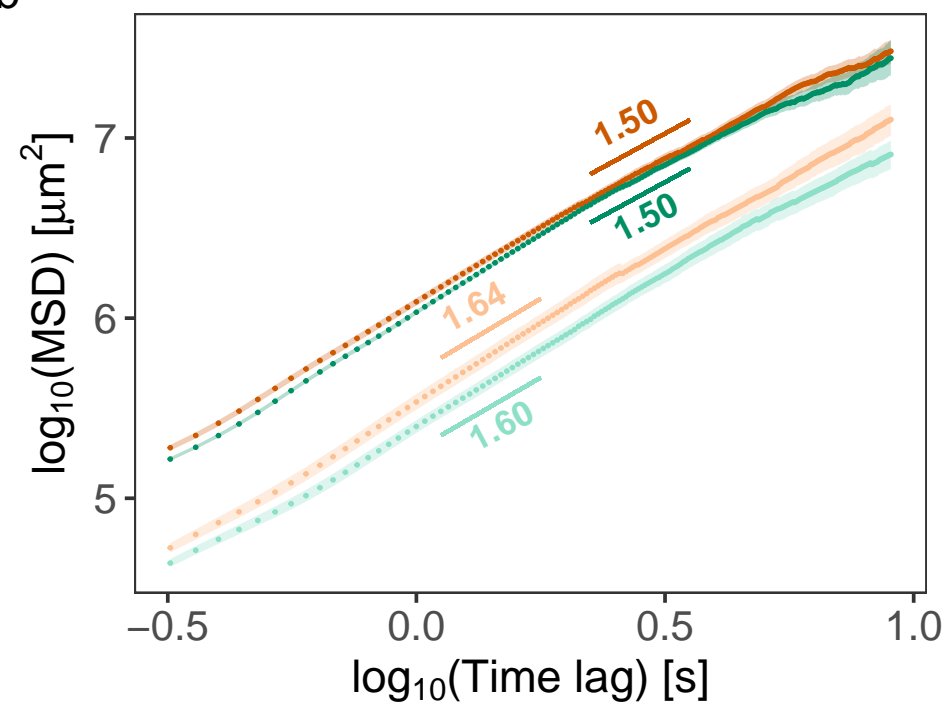

- Fast predator in monoculture

- Fast predator in mixed culture
Slow predator in monoculture Slow predator in mixed culture 


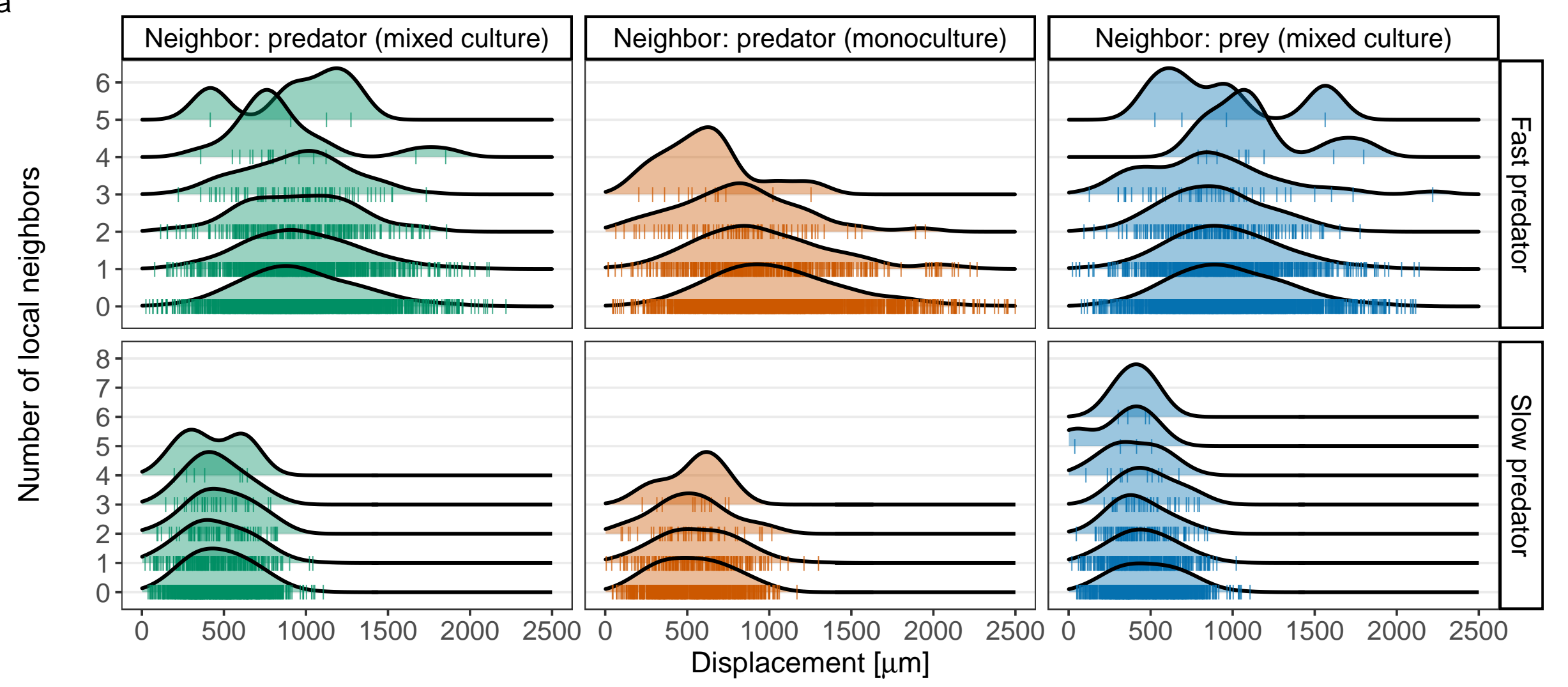

b

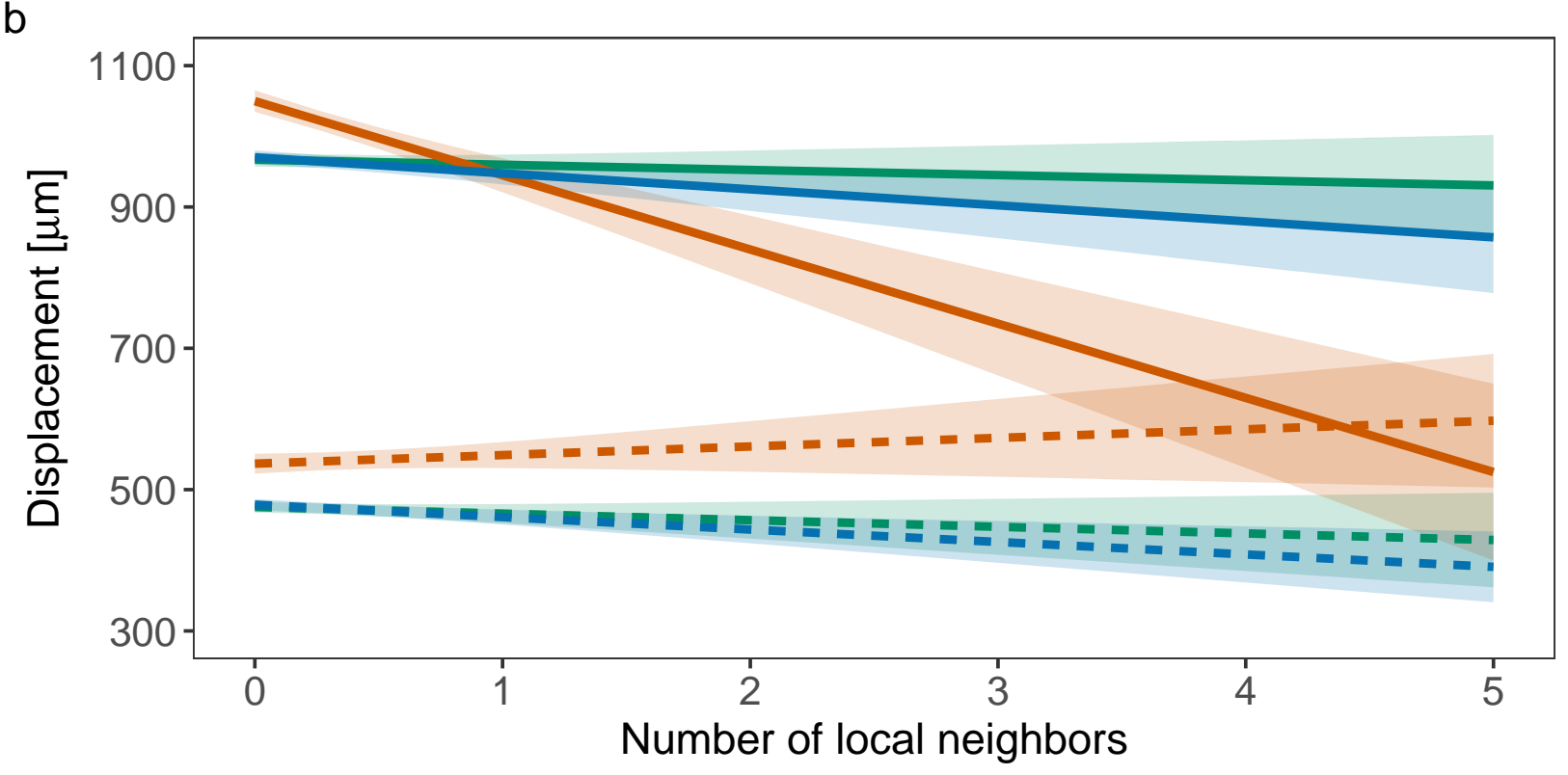

Swimming regime

- Fast

Neighbor type

- Predator (mixed culture)

- Predator (monoculture)

- Prey (mixed culture)

Number of local neighbors 


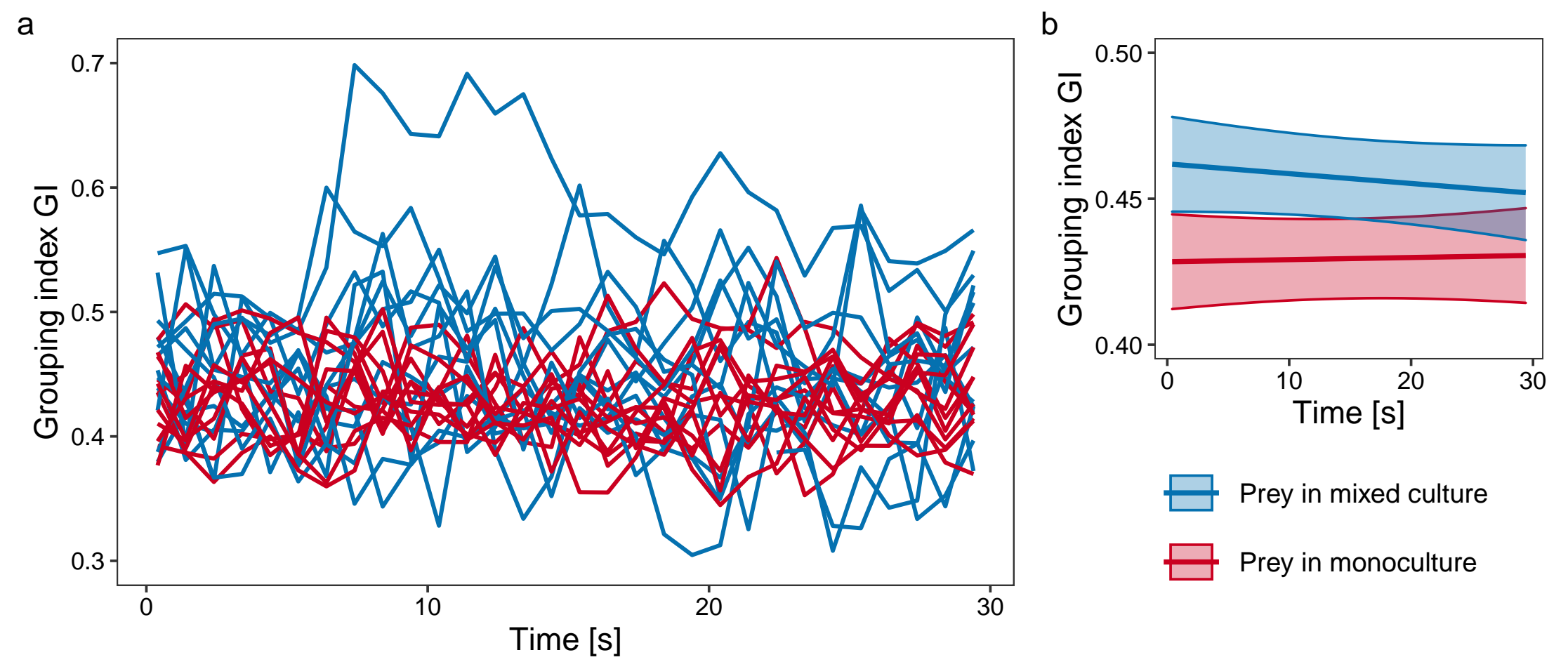

